\title{
Sobre la observación participante en la Escuela de Chicago: un análisis de las monografías fundacionales ${ }^{1}$
}

\author{
Javier Santos, Juan Ignacio Piovani y \\ María Eugenia Rausky
}

\begin{abstract}
Resumen
La idea de una Escuela Sociológica de Chicago en el período de entreguerras ha resultado persistente -en el marco de una interpretación que podríamos considerar clásica- bajo el supuesto lugar privilegiado que ésta tuvo en el desarrollo de los métodos cualitativos de investigación social (en general) y de la observación participante (en particular). Sin embargo, algunos de los autores que han avanzado en la reconstrucción de la historia de los métodos de investigación sociológica han cuestionado que se trate de métodos cualitativos en el sentido actual, presentando así una interpretación revisionista de la Escuela y de sus aportes metodológicos.

Teniendo en cuenta esta tensión entre interpretaciones clásicas y
\end{abstract} revisionistas, en este artículo nos proponemos caracterizar las prácticas de investigación de campo (fieldwork) desplegadas en las monografías chicaguenses (tesis doctorales) que luego serían tomadas como hitos fundacionales de la observación participante sociológica por parte de los mentores de la interpretación clásica.

Estas prácticas observacionales aplicadas en la investigación empírica de Chicago son interpretadas a partir de dos dimensiones: por una parte desde el punto de vista instrumental/operativo (técnico), recurriendo a la tipología desarrollada por Gold (1958) en su célebre artículo sobre los roles de observación y participación. Por otra parte, desde el punto de vista teórico-epistemológico, teniendo en cuenta su relación con supuestos objetivistas/cientificistas o interpretativos/cualitativos.

Esta doble dimensión del análisis (epistemológico/técnico) permite mediar entre las versiones clásicas y revisionistas de la Escuela de Chicago, destacando la aplicación pionera en este contexto de técnicas análogas (al menos superficialmente) a la moderna observación participante (aspecto técnico), pero fundamentadas en general a partir de postulados objetivistas y cientificistas, y no en el marco de las concepciones interpretativas que en la actualidad dan sustento a la investigación cualitativa (aspecto epistemológico).

Palabras clave: Escuela de Chicago, Metodología cualitativa, Obervación Participante

1 Una versión preliminar de este artículo fue presentada como ponencia en el XXVII Congreso Internacional de la Asociación Latinoamericana de Sociología (ALAS), Buenos Aires, 31 de agosto - 4 de septiembre de 2009. 
Sobre la observación participante en la escuela de Chicago. Un análisis de las monografías fundacionales

\begin{abstract}
The idea of a Sociological School of Chicago during the interwar period has been persistent -from a perspective that could be considered classical-, under the assumption that it had a special place in the development of qualitative methods of social research (in general) and of participant observation (in particular). However, some authors that focused on the history of sociological research methods have contested the idea that it developed qualitative methods in the modern sense, presenting a revisionist interpretation of the School and its methodological contributions.

Given this tension between traditional and revisionist interpretations, this paper examines the practices of field research (fieldwork) portrayed in the Chicagoans' monographs (doctoral thesis) that were later taken as foundational landmarks of sociological participant observation by the mentors of the above-mentioned classical interpretation.

These observational practices applied in empirical investigations conducted by Chicagoans are analyzed from two dimensions: on one hand from the instrumental/operational (technical) standpoint, using the typology developed by Gold (1958) in his famous article on the roles of observation and participation. On the other hand, from the theoreticalepistemological perspective, taking into account their relationship with objectivist/scientistic or either interpretive/qualitative assumptions.

This double dimension of analysis (epistemological/technical) allows to mediate between the classical and revisionist versions of the Chicago School, highlighting its pioneering application of research techniques at least superficially similar to modern participant observation (technical aspect), but based on objectivist and scientistic principles, and not on the kind of interpretative concepts that currently support qualitative methodology (epistemological aspect).
\end{abstract}

Key words: Chicago School, Qualitative Methodology, Participant Observation 


\section{Introducción}

La idea de una Escuela Sociológica de Chicago en el periodo de entreguerras ha resultado persistente -en el marco de una interpretación que podríamos considerar clásica- bajo el supuesto lugar privilegiado que ésta tuvo en el desarrollo de los métodos cualitativos de investigación social, en general, y de la observación participante, en particular (Taylor y Bogdan, 1986; Denzin y Lincoln, 1994; Bryman, 2001; Forni, 1993; Vasilachis, 1993).

No obstante, este destacado rol en el pasado disciplinar sólo le fue conferido/reconocido tardíamente -hacia mediados de la década de 1960-, cuando emerge un conjunto de obras que operan un proceso de reconstrucción/revisión de la tradición sociológica, cuya historia había sido objeto de interés por parte de los sociólogos estadounidenses desde larga data (Sica, 2007), pero sin que se hubiera identificado con anterioridad la especificidad de la Escuela de Chicago.

Existe un consenso difundido entre los especialistas acerca de que la serie The Heritage of Sociology, creada en 1964 por Morris Janowitz en la editorial de la Universidad de Chicago, jugó un papel crucial para posicionar la relevancia de la tradición chicaguense en la historia de la disciplina, principalmente con las obras de Robert Lee Faris (1967) y James Carey (1975).

El libro de Faris, Chicago Sociology 1920-1932, fue el primero de la serie con un perfil socio-histórico. A partir de una reconstrucción del devenir del Departamento de Sociología entre la década de 1920 y principios de la década de 1930, Faris sostiene la existencia de una Escuela, poniendo el énfasis en la centralidad de figuras como William I. Thomas y Robert E. Park, y en las tradiciones de la Ecología Humana, la Psicología Social y los Estudios Empíricos Urbanos. James Carey, por su parte, publica en 1975 Sociology and Public Affairs: The Chicago School, un trabajo que comparte las líneas generales de la obra de Faris, pero destaca también el rol que jugaron las conexiones entre la Escuela de Chicago y los asuntos públicos. 
Sobre la observación participante en la escuela de Chicago. Un análisis de las monografías fundacionales

Hacia fines de la década de 1970 la Escuela de Chicago se encontraba ya posicionada y enmarcada como objeto historiográfico, caracterizado por un espacio y un tiempo delimitados, y por un conjunto de sociólogos que, entre 1915 y 1935, se destacaron por: a) su rol innovador en el desarrollo de un estilo de investigación social de carácter empírico y sistemático focalizado en las problemáticas urbanas; $b$ ) la generación de nuevas estrategias para la recolección de datos, como la observación participante y, c) su énfasis en la producción de información de primera mano.

Sin embargo, durante la década de 1980 aparece un cambio de perfil en los estudios sobre la tradición chicaguense. En este proceso, la obra de Martin Bulmer, The Chicago School of Sociology (1984), jugó un papel clave: su extenso trabajo de reconstrucción histórica significó un gran aporte al conocimiento de la Escuela de Chicago, en especial por su detallado análisis de los aspectos institucionales, pero también constituyó un ataque consistente a las visiones tradicionales que la asociaban unívocamente con el enfoque cualitativo, ${ }^{2}$ dando origen así a una interpretación que, aunque no carente de matices, hemos denominado revisionista. $^{3}$

2 Tanto M. Bulmer, L. Harvey, Hammersley y Young y England han afirmado que es erróneo asociar unívocamente la lógica cualitativa con la Escuela de Chicago, puesto que conlleva negar la existencia de la línea cuantitativa en este mismo espacio institucional. Según ellos, estas afirmaciones remiten a descuidos, engaños o mitos generados por visiones antipáticas frente a lo cuantitativo. Sin embargo, no se trata de una discusión cerrada: en el último tiempo han aparecido voces en defensa de la asociación del Departamento con el abordaje cualitativo, como el caso de David Nock.

3 Desde el revisionismo metodológico destacan los aportes de Anthony Oberschall, The Establishment of empirical Sociology (1972); Michael Bodemann, A problem of Sociological Praxis (1978); Jeff Verhoeven, An interview with Erving Goffman (1993); Martyn Hammersley, The Dilemma of Qualitative Method (1989); Jennifer Platt, The development of the "Participant Observation" method in sociology (1983), Weber's Verstehen and the History of Qualitative Research (1985), Chicago Methods and the first hand data (1994), Research Methods and the Second Chicago School (1995), A History of Sociological Research Methods in America (1996) y Chicago Methods: reputations and realities (1998); Martin Bulmer, The Chicago School of Sociology (1984); Lee Harvey, Myths of the Chicago School of Sociology (1987); Jean-Michel Chapoulie, Everett C. Hughes and the Development of Fieldwork in Sociology (1987) y La tradition sociologique de Chicago (2001); Norman Denzin, Stanley and Clifford: Undoing an Interactionist Text (1995) y David Nock, The Myth about "Myths of the Chicago School". Evidence from Floyd Nelson House (2004). 
En la disputa entre interpretaciones clásicas y revisionistas de la Escuela, uno de los aspectos más controvertidos ha sido -justamente- el papel que ésta tuvo en el desarrollo de los métodos cualitativos de investigación. En particular, se ha escrito profusamente sobre: 1) los orígenes de la observación participante (supuestamente chicaguenses) y, 2) su relación con las perspectivas teórico-epistemológicas interpretativas.

En cuanto a la primera cuestión, Jennifer Platt (1983; 1985) -autora de referencia del punto de vista revisionista- se ha diferenciado de la perspectiva clásica al sostener el carácter "mítico" de su origen en dicho espacio institucional. Esta conclusión se basa en tres argumentos: a) en la discusión metodológica anterior a la Segunda Guerra Mundial el acceso a los significados no estaba asociado con la participación; b) la observación participante no fue aplicada en la Escuela de Chicago en el sentido actual ${ }^{4}$, sino con el fin de acceder a datos "objetivos" (muchas veces cuantificables); c) el término 'observación participante', introducido hacia 1924, se refería en ese contexto a una cosa distinta, y aludía al uso de sujetos nativos en calidad de informantes del investigador.

El otro aspecto de relevancia que se ha abordado con relación al tema es el aparente nexo entre la observación participante -y los métodos cualitativos en general- con la corriente interpretativa de la sociología y, en particular, con la idea weberiana de verstehen. Platt (1985) ha argumentado que no existe evidencia acerca de que los aportes teóricos y epistemológicos de Weber hayan tenido la suficiente influencia en etapas formativas del desarrollo de los métodos cualitativos de investigación en EE.UU. Si bien es común el reconocimiento de la influencia de Weber en los métodos cualitativos de investigación, la visión clásica de la Escuela

4 En la actualidad, en la literatura metodológica se define a la observación participante como una técnica de recolección de información cuya función es garantizar el acceso privilegiado a los significados que los actores construyen (y le asignan a su mundo) a través del hecho de observar "en directo" y compartir experiencias en el ambiente que se estudia (Platt, 1982). 
Sobre la observación participante en la escuela de Chicago. Un análisis de las monografías fundacionales

de Chicago pareciera otorgarle un lugar central en los orígenes de la observación participante sociológica que -de acuerdo con la perspectiva revisionista- no le corresponde. ${ }^{5}$

Teniendo en cuenta esta tensión entre interpretaciones clásicas y revisionistas, en trabajos anteriores hemos analizado distintos aspectos vinculados con los desarrollos metodológicos de la Escuela de Chicago. Más específicamente, hemos indagado acerca de los usos y sentidos de los términos metodológicos cualitativos en los artículos publicados en su revista -el American Journal of Sociology (AJS) - y acerca del significado de 'observación partici-

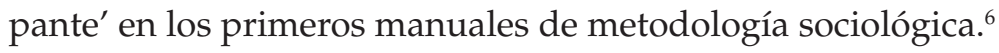

En la misma línea de este último trabajo, en este artículo nos proponemos caracterizar las prácticas de investigación de campo (fieldwork) desplegadas en las monografías (tesis doctorales) que luego serían tomadas como hitos fundacionales de la observación participante sociológica por parte de los mentores de la interpretación clásica de la Escuela.

Estas prácticas observacionales aplicadas en la investigación empírica llevada a cabo por estudiantes de doctorado de la Escuela son interpretadas en nuestra propuesta a partir de dos dimensiones. Por un lado, desde el punto de vista instrumental/ operativo (técnico), recurriendo a la tipología desarrollada por Gold (1958) en su célebre artículo sobre los roles de observación

5 Sobre las bases teóricas y filosóficas que han dado fundamento a la metodología de la Escuela de Chicago y a su desarrollo del fieldwork también se destacan los aportes de Cefai (2001), Cortese (1995), y Hammersley (1981). Estos autores señalan, en general, la influencia de corrientes como el Pragmatismo y el Interaccionismo Simbólico, y de autores como Dewey, Simmel y Cooley.

6 El primero de dichos trabajos fue presentado en 2007 en el marco del XXVI Congreso de ALAS. El segundo fue presentado en 2008 en las V Jornadas de Sociología de la UNLP y I Encuentro Latinoamericano de Metodología de las Ciencias Sociales.

7 Gold (1958) desarrolla una tipología de los diferentes roles que se pueden asumir en relación con la observación y la participación: a) participante completo / inmerso; b) participante como observador; c) observador como participante y d) observador pleno. En un extremo se encuentra el participante completo / inmerso, quien ocultando su rol de investigador se transforma en un miembro del grupo estudiado sin interrumpir sus actividades cotidianas. El participante como observador, en cambio, se inserta como miembro de un grupo para estudiarlo, pero informa a los sujetos investigados sobre su rol investigativo. La posición de observador como participante 
y participación. Por el otro, desde el punto de vista teórico-epistemológico, teniendo en cuenta su relación con supuestos objetivistas/cientificistas o interpretativos/cualitativos.

Con esta doble dimensión analítica (epistemológica / técnica) se aspira a establecer posibles mediaciones entre las versiones clásicas y revisionistas de la Escuela de Chicago, intentando reconstruir la aplicación pionera de técnicas análogas -al menos superficialmente- a la moderna observación participante (aspecto técnico), aunque posiblemente fundamentadas en postulados generales de carácter objetivista y cientificista, y no en el marco de las concepciones interpretativas que en la actualidad dan sustento a la investigación cualitativa (aspecto epistemológico).

\section{La observación participante en el contexto de las monografías fundacionales ${ }^{8}$}

Platt (1983) destaca tres monografías (tesis doctorales) en las que se reportan investigaciones empíricas en el marco de las cuales habría tenido lugar la implementación originaria de la observación participante en la Escuela de Chicago, razón por la cual las hemos denominado "fundacionales" a los efectos del análisis que proponemos en este trabajo:

- Nels Anderson (1923): The Hobo: The Sociology of the Homeless Man

- Frederic Thrasher (1927): The Gang: A Study of 1313 Gangs in Chicago

- Paul Cressey (1932): The Taxi-Dance Hall: A Sociological Study in Commercialized Recreation and City Life

implica una observación más bien formal, y el investigador suele limitarse en este caso a la realización de entrevistas. Finalmente, el observador pleno no interactúa con los sujetos estudiados, y observa desde una posición que queda oculta a la vista de los sujetos investigados.

8 Esta sección se basa en una reelaboración abreviada del punto 6.2 de la tesis de J. Santos, dirigida por J. I. Piovani: “La observación participante y la Escuela de Chicago", presentada y defendida en el año 2008 en el marco de la Maestría en Metodología de la Investigación Social (UNIBO-UNTREF). 
Sobre la observación participante en la escuela de Chicago. Un análisis de las monografías fundacionales

The Hobo ${ }^{9}$ : The Sociology of the Homeless Man $(1923)^{10}$ es un trabajo de investigación desarrollado por Nels Anderson acerca de la población de trabajadores migrantes que, sin residencia fija, se trasladaba de un lugar a otro en busca principalmente de oportunidades laborales.

El interés de Anderson por la temática Hobo está íntimamente ligado a su historia personal y familiar: durante su niñez vivió situaciones de extrema precariedad mientras su padre se desplazaba en búsqueda de trabajo. En la adolescencia abandonó el hogar y comenzó un derrotero incierto por distintos empleos temporales. Esta experiencia de vida, como observador participante nativo, facilitó la toma de contacto con su población de estudio instalándose en la habitación de un hotel obrero en una zona de Chicago conocida como Hobohemia. Durante un año recolectó información compartiendo la cotidianidad de la vida de Hobo, ya sea permaneciendo durante períodos extensos en distintos puntos de la zona, manteniendo conversaciones casuales, durmiendo al aire libre, viajando clandestinamente en trenes y/o buscando y realizando trabajos estacionales que le permitieran estar en contacto permanente con los Hobos.

Si bien es extensa, la obra original carece de referencias metodológicas detalladas pues, como comenta el mismo autor en la introducción a la Phoenix Edition de 1961, sus conocimientos acerca de "cómo" hacer la investigación eran entonces limitados:

La guía que recibí en la Universidad de Chicago por parte de los profesores Robert E. Park y Ernest Burgess fue, en su mayoría, indirecta. La única instrucción de Park fue, 'anota sólo lo que veas, escuches y conozcas, como un periodista de Diario' (Anderson, 1923/1967: xii, traducción propia).

9 Hobo es un término de origen incierto introducido en EEUU entre 1885 y 1890 para referirse al trabajador golondrina (Webster's Encyclopedic Unabridged Dictionary of the English Language, 1996).

10 Esta fue la obra inaugural de la serie editorial que cristalizaría y difundiría la manera de hacer sociología en la Escuela de Chicago: The Sociological Series. 
En efecto, la formación en investigación que se ofrecía en el célebre seminario de Fieldstudies a cargo de Park y Burgess carecía de la sofisticación teórica y técnica que conocemos en la actualidad. En general los docentes se limitaban a impulsar a los estudiantes a entrar al campo a través del contacto directo e implementando entrevistas informales iniciales. Sin embargo, no existían indicaciones metodológicas fuertemente estructuradas.

La perspectiva investigativa de Anderson, no obstante, no privilegiaba necesariamente a la observación participante: en cierta medida, la recolección de datos de primera mano a través de observaciones, conversaciones y participación en las actividades cotidianas con los Hobos fue una forma pragmática de superar los obstáculos para la aplicación de cuestionarios semiestructurados, que de haber sido viable -como él mismo reconoce- hubiera sido su preferencia. Por otra parte, también recopiló información documental (estadísticas, documentación oficial, publicaciones, etc.) que utilizó como parte de su fundamentación en el análisis.

Para el autor, la observación participante no implicó tanto el desarrollo consciente de un rol, en el sentido de "hacerse pasar por", sino más bien el hecho de revisitar un estilo de vida que le había sido propio. Pero independientemente del grado de autoconsciencia en la asunción del rol, la posición jugada por Anderson en el campo guarda directa relación con las prácticas típicas de lo que actualmente conocemos como "Participante Completo" (Gold, 1958), ya que mantuvo encubiertos sus objetivos y su papel de investigador, y esto sí lo hizo conscientemente, buscando acceder sin "distorsiones" a las historias y concepciones de vida de los Hobos.

Anderson tenía en mente el requerimiento de acceder a toda aquella información que permitiera dar cuenta de la complejidad de la temática Hobo en Chicago, concibiendo su indagación, como el resto de los investigadores de Chicago, en términos del Estudio de Caso. En este contexto, la observación participante (expresión de la que él nunca había oído hablar en los años en que realizó su 
Sobre la observación participante en la escuela de Chicago. Un análisis de las monografías fundacionales

trabajo de campo) era una estrategia, entre otras, para acceder a "datos objetivos" relativos a su objeto de estudio.

The Gang: A Study of 1313 Gangs in Chicago (1927) es una monografía realizada por Frederic Thrasher sobre el surgimiento y las características del fenómeno masivo de las bandas o pandillas juveniles en la ciudad de Chicago. Enmarcado en el campo de la naciente ecología humana y urbana, el abordaje subraya la interacción entre las dimensiones espaciales y del comportamiento individual en el ámbito de una ciudad en continuo desarrollo y cambio.

De la obra se desprende que el trabajo de campo insumió cerca de siete años y que se recolectó una diversidad de datos, incluyendo: información censal, registros de juzgados y de agencias públicas, información periodística y documentos personales de miembros de bandas. Además, se llevaron a cabo observaciones personales y entrevistas, y se recurrió a observaciones realizadas por terceros en distintos contextos.

En el marco de esta estrategia de investigación ecléctica, la observación participante no sólo no adquiere un estatus especial, sino que por el contrario los datos derivados de ella tienen un "valor" relativo menor que el asignado a otras fuentes. Por otra parte, en las citas que son resultado de prácticas observacionales directas ${ }^{11}$ no hay precisiones metodológicas iluminadoras. Sin embargo, un artículo publicado por Thrasher un año después de la monografía aquí analizada, titulado How to Study the Boys' Gang in the Open ${ }^{12}$, aporta indicios clave con relación a los sentidos y funciones que le asigna a la observación participante:

Uno no puede entrar en una banda y hacer preguntas. Los chicos en este ambiente sospechan bastante de tal procedimiento. El primer problema es seleccionar un grupo, y a través de un muy cuidadoso plan, establecer rapport. Un método es hacerse

11 Caso 27 y Caso 49.

12 Thrasher, F. (1928). Journal of Educational Sociology 1, 5: 244-254. 
conocido gradualmente con algunos miembros de la banda sin parecer particularmente interesado en ellos. Pasar cada día, parar para observarlos ocasionalmente y bromear de vez en cuando, haciendo algún pequeño favor como ofrecer una tabla para una patineta, gradualmente aprender y llamarlos por sus nombres, emplear alguno de vez en cuando como ayuda en alguna pequeña cuestión como trasladar paquetes, etc. Este procedimiento requiere varias semanas pero, en algún momento, el investigador se vuelve lo suficientemente conocido para que el grupo confie en él y [...] pueda sugerir alguna actividad como ir a un espectáculo, armar un juego, tomar una excursión o algún proyecto similar que motive a los chicos. Habiendo participado en tal empresa con los chicos, las oportunidades de conversación y observación serán mayores (Thrasher, 1928: 244, traducción propia).

Como en The Hobo, la implementación de la observación participante está íntimamente relacionada con el reconocimiento de las dificultades de aplicación de otras estrategias. Thrasher parte de la necesidad de abordar las pandillas sin la mediación de agencias sociales, con el fin de evitar obstrucciones en la recolección de información; pero su estrategia no se enmarca en una perspectiva "naturalista" para el estudio de las bandas de chicos no institucionalizados en sus contextos cotidianos. Por el contrario, la observación participante se da en el marco de "situaciones artificiales" generadas por el propio investigador, una vez adquirido cierto grado de confianza con los chicos, con el fin de favorecer instancias puntales de interacción y, especialmente, permitir la realización de algún tipo de entrevista no disruptiva. En este sentido, pareciera no ser relevante para el autor su intervención y modificación de las dinámicas grupales en su interés por conocer los grupos.

No hay en la obra de Thrasher una reflexión sistemática acerca de su rol de observador participante. Su preocupación metodológica más explícita se vincula con las estrategias que mejor 
Sobre la observación participante en la escuela de Chicago. Un análisis de las monografías fundacionales

garanticen el establecimiento de rapport y la generación de la confianza necesaria para poder acceder a la información requerida en la investigación (a través de entrevistas individuales o grupales). A diferencia de lo que hace Anderson, Thrasher no plantea la posibilidad de "sumergirse" en el ámbito de una banda y participar de su cotidianidad (de manera encubierta o no). Está claro, de todos modos, que la diferencia de edad (entre él y los chicos estudiados) presenta un obstáculo insalvable para ello. En este mismo sentido debe reconocerse la imposibilidad fáctica de lograr integrarse simultáneamente en la cotidianidad de las más de mil bandas que constituían su marco de interés.

El tipo de práctica observacional del autor difícilmente pueda encuadrarse en la perspectiva de un observador que asume deliberadamente este papel y que participa de manera "naturalista" en la vida comunitaria que estudia. Thrasher desplegó un tipo de rol más asociado con la estrategia del "observador como participante" (Gold, 1958), porque tendió a limitar el trabajo de campo a la entrevista, con actividades de observación más bien formales, sin que se generen las condiciones para una observación/participación informal y naturalista a lo largo del tiempo (Piovani, 2007).

The Taxi-Dance Hall: A Sociological Study in Commercialized Recreation and City Life (1932) es un trabajo de investigación realizado entre 1925 y 1929 por Paul Cressey sobre los salones de baile pagos aparecidos a inicios del siglo XX en Chicago. Los TaxiDance Halls eran ámbitos de esparcimiento en los que mujeres contratadas bailaban a cambio de una remuneración. El público que concurría a estos salones estaba constituido principalmente por inmigrantes proletarios de paso por la ciudad (Buchan Crook, 1934). Para la opinión pública de la época estos lugares eran sólo una fachada que encubría el ejercicio de la prostitución.

De acuerdo con Ernest Burgess, autor de la introducción de la monografía, se trata de una indagación sobre el mundo del Taxi-Dance Hall desde una perspectiva naturalista, ecológica e 
imparcial. A pesar de compartir el enfoque metodológico típico de la Escuela de Chicago, en el marco del cual se buscaba recurrir a diferentes fuentes de datos para la construcción del Estudio de Caso, la carencia de datos secundarios disponibles (por ejemplo estadísticas) y la imposibilidad de acceder a documentos personales a partir de estrategias como las entrevistas, llevaron al autor a implementar prácticas de observación participante que hacen de su indagación un caso diferencial entre las monografías fundacionales. Por otra parte, se encuentra ya en el Prefacio de la obra la enunciación explícita de algunas de las premisas metodológicas fundamentales que guiaron la investigación:

Se enviaron observadores a los Taxi-Dance Halls. Se los instruyó para mezclarse con los otros y transformarse en parte de este mundo social cuanto éticamente fuera posible. Se les pidió que observaran y que se mantuvieran lo más ajustados posible los registros del comportamiento y de las conversaciones [...] Cada observador fue seleccionado por su experiencia pasada, su entrenamiento, y sus habilidades especiales. Estos investigadores hicieron posible reunir material de caso [...] mucho más variado de clientes y taxi-dancers de lo que hubiera conseguido cualquier otra persona [...] Además, los reportes de los diferentes observadores sobre los contactos con el mismo individuo hicieron posible un control sobre la consistencia de los documentos obtenidos (Cressey, 1932: xvii-xviii, traducción propia).

Como se deriva claramente de la cita precedente, la observación no fue únicamente desarrollada por el autor sino también por varios asistentes. En tanto que la observación participante era considerada como una técnica que permitía acceder a datos a los que de otra forma hubiera sido imposible acceder, la multiplicidad de observadores garantizaba mayor acceso, mayor volumen de información significativa y mayores posibilidades de control de consistencia de la información recolectada. 
Sobre la observación participante en la escuela de Chicago. Un análisis de las monografías fundacionales

El rol de los observadores no era arbitrario; por el contrario, implicaba conciencia de acción y reflexión sobre las prácticas:

Los investigadores funcionaron como extraños anónimos y conocidos casuales. Fueron capaces, así, de obtener este material sin encontrar las inhibiciones y resistencias usualmente encontradas en las entrevistas formales (Cressey, 1932: xviii, traducción propia).

Si bien en la monografía no se encuentran muchos más detalles sobre la perspectiva metodológica, para dar cuenta de la particular posición de Cressey se puede recurrir a un artículo escrito por él en 1927, aunque publicado recién en 1983 en la revista Urban Life. Realizado contemporáneamente al trabajo de campo sobre el Taxi-Dance Hall, el autor explicita allí su reflexión acerca del rol del observador, teniendo como principal referente a Georg Simmel. ${ }^{13}$ Se trata de una de las primeras presentaciones chicaguenses sobre el rol de observador en la investigación sociológica (Bulmer, 1983) y pone de manifiesto cierto grado de reflexión metodológica respecto de la práctica de la observación participante.

Cressey plantea tres tipos de observación social. El primero refiere al contacto con los íntimos (intimates). El investigador puede hacer uso de sus conexiones relacionales inmediatas para la indagación como lo hiciera Charles Cooley, por ejemplo, observando a sus hijos para dar cuenta de los comportamientos de los niños. El segundo tipo se da cuando el investigador asume el rol de extraño (stranger), en los términos planteados por Simmel. El investigador social, en cuanto Sociological Stranger, combina la cercanía (estar físicamente presente) con la distancia (cultural), habilitando de este modo un desapego virtuoso en la comprensión del mundo en estudio. El tercer tipo se vincula con la situación de anonimato: los contactos entre el investigador y los miembros del grupo analizado se dan en la forma de "extraños anónimos"

13 Simmel era de lectura obligatoria en el curso de Fieldstudies y uno de los autores europeos más referenciados en el manual Intoduction to the science of sociology de Park y Burgess. 
o "conocidos casuales" (Anonymous Stranger). Esta última estrategia, a la que recurrirá Cressey, no era nueva en la práctica de la investigación en Chicago aunque sí poco frecuente.

Según el autor, el rol de Anonymous Stranger permitía establecer relaciones con carácter transitorio, implementar un tipo intensivo de acceso a la información y mantener un contacto informal, casual y desinhibidor. Por otra parte, parecía más adecuado para el estudio de los Taxi-Dance Halls porque permitía superar las limitaciones de la estrategia del Sociological Stranger, que presentaba serios obstáculos en el acceso a la información (como consecuencia de la negativa de los dueños de los salones para admitir el trabajo de los investigadores $\mathrm{y}$, tal vez, por los efectos reactivos de la presencia "reconocida" de ellos). Para Cressey, el Anonymous Stranger debía asumir una posición no moralista y desprejuiciada; la clave estaba en encontrar el momento adecuado para establecer una conversación informal en un lenguaje compartido, en búsqueda de relatos significativos.

\section{Conclusiones}

La reconstrucción histórica de la observación participante en Sociología ha dado lugar a una versión arquetípica que se produce y reproduce en los procesos de socialización secundaria de los especialistas, a través de cursos sistemáticos y de manuales de metodología. Esta versión le asigna a la Escuela de Chicago un lugar fundacional tanto en los aspectos técnicos como teóricoepistemológicos de la observación participante.

En contraposición, especialmente a partir de mediados de la década de 1980, y "liderada" por Platt, se articuló una versión revisionista que, en sus variantes más extremas, le ha negado a la Escuela todo lugar en el desarrollo de la observación participante, ya sea desde el punto de vista teórico-epistemológico o desde el punto de vista técnico.

Como se ha indicado en la introducción, este trabajo apuntaba en cierto sentido a mediar entre ambas versiones -la clási- 
Sobre la observación participante en la escuela de Chicago. Un análisis de las monografías fundacionales

ca y la revisionista-, revisitando algunas fuentes primarias -las monografías fundacionales- con el fin de sopesar los distintos argumentos y proponer, si esto fuera posible, una nueva lectura acerca del lugar que tuvo y las características que adquirió la observación participante en el marco de las investigaciones empíricas del período clásico de Chicago. En este sentido, el análisis propuesto permite destacar lo siguiente:

Aunque en ninguno de los trabajos aparece la expresión 'observación participante' (tal vez en parte porque en la década de 1920, cuando ésta fue introducida, remitía más bien a la idea actual de "informante clave"), es evidente que existieron prácticas (en su sentido técnico) que no deben desconocerse en cuanto esfuerzos observacionales directos.

Resulta claro que tanto Anderson como Cressey, y en menor medida Thrasher, pusieron en juego alguna forma de observación participante. En efecto, con diferencia en la forma y el tiempo de acercamiento, los autores tomaron contacto inmediato con las temáticas de interés a través de grupos de sujetos en sus "ambientes naturales". Estas formas de contacto implicaron cierta reflexión y decisión consciente acerca del rol de observador: todos debieron tomar una decisión, por ejemplo, sobre mantener encubierta su identidad y objetivos a los efectos de facilitar el acceso a la información. Sin embargo, la observación participante se utilizó, en gran medida, con un sentido puramente pragmático, debido a las limitaciones que presentaban estos casos particulares para el empleo de otras técnicas que los autores juzgaban científicamente más adecuadas.

Anderson implementó un estilo de observación participante cercana a la del "sentido nativo", es decir, la práctica de alguien que pertenece al grupo y que participa "naturalmente" en él, pero que a partir del extrañamiento y el distanciamiento busca convertirlo en objeto de indagación. Por otra parte, su experiencia es superficialmente análoga a la del "Observador Completo" (Gold, 1958) en tanto no se revelan la identidad ni los objetivos del investigador. 
Thrasher implementó un estilo de "Observador como Participante", realizando fundamentalmente entrevistas grupales y compartiendo actividades acotadas (no naturalistas, y por lo tanto más limitadas en su validez ecológica) con los miembros de las pandillas en diferentes oportunidades.

Como la de Anderson, la observación participante de Cressey (y su equipo) también fue encubierta. Pero no puede atribuírsele un "sentido nativo" en tanto que los Taxi-Dance Halls no constituían su espacio cotidiano de sociabilidad (como sí lo había sido el Hobo en el caso de Anderson, al menos durante su infancia y juventud). Este autor es tal vez el primero que concibe a la observación participante como el desarrollo de un rol profesional de investigación. De los roles posibles conceptualizados por Gold (1958), sus prácticas cumplen todos los requisitos del "Participante Completo". ${ }^{14}$ La figura de Cressey adquiere, en este sentido, el status de referente distintivo por la mayor sistematicidad y madurez metodológica de su reflexión en torno del rol del observador participante y su propuesta del extraño anónimo.

Pero si se tiene en cuenta la dimensión teórico-epistemológica, debemos reconocer que la observación participante -tal como se la define en la actualidad- se enmarca en un estilo de investigación cualitativo-interpretativo y no remite únicamente a los aspectos técnicos de una forma de contacto directo con actores sociales "situados" que posibilite "recolectar" datos sobre determinado fenómeno.

Desde este punto de vista, debemos coincidir con Platt en que los autores analizados estaban principalmente preocupados por la recolección de información "objetiva" y no tenían la intención de acceder a significados en el sentido interpretativo actual.

14 Es interesante hacer notar que su defensa de la participación completa en el marco de la investigación se hace en términos análogos a los de Homan (1980), obra considerada a la vez clásica y pionera en este tema, sólo que casi 50 años antes: la única justificación posible para el encubrimiento radica en el riesgo certero de que la revelación de la identidad y de los objetivos del investigador impida el acceso a los fenómenos de interés sin que éstos resulten significativamente alterados. 
Sobre la observación participante en la escuela de Chicago. Un análisis de las monografías fundacionales

Esta concepción de la observación participante en cuanto medio (técnico) de recolección de datos objetivos es uno de los argumentos más fuertes en contra del reconocimiento de las prácticas de campo de la Escuela de Chicago como origen de la moderna observación participante cualitativa. En este sentido, resulta pertinente recordar que recién en el marco de lo que se conoce como Segunda Escuela de Chicago (a partir de la década de 1950), con los aportes de Howard Becker y Everett Hughes, la observación participante asumiría en este contexto institucional un sentido más contemporáneo asociado a las perspectivas interpretativas.

Sin embargo, si bien es importante ratificar el carácter mítico del origen de la observación participante en este período clásico de la Escuela de Chicago, no resulta igualmente justo negarle todo valor innovador a sus prácticas metodológicas. Las formas de observación directa desarrolladas por los autores analizados, aunque no pueden considerarse el origen de la visión actual sobre la observación participante, deberían ser tenidas en cuenta -al menos-como importantes antecedentes.

\section{Bibliografía}

ANDERSON, NELS (1923/1967). The Hobo. The sociology of the homeless man. Chicago, Phoenix.

BODEMANN, MICHAEL (1978). "A problem of social praxis: The case for interventive observation in fieldwork", en Theory and Society, 5, 3, pp: 387-420.

BRYMAN, ALAN (2001). Ethnography. London, Sage Publications.

BUCHAN CROOK, EVELYN (1934). "Reseña de Paul G. Cressey The TaxiDance Hall. A Sociological Study in Commercialized Recreation and City Life", en American Journal of Sociology, 40, 1, pp: 123-124.

BULMER, MARTIN (1983). "The Methodology of The Taxi-Dance Hall: An Early Account of Chicago Ethnography from the 1920s", en Journal of Contemporary Ethnography. 12, 1, pp: 95-120.

BULMER, MARTIN (1984). The Chicago School of Sociology: Institutionalization, Diversity, and the Rise of Sociological Research. Chicago, University of Chicago Press. 
BURGESS, ROBERT (1996). In the field: An introduction to field research. Londres, Routledge.

CAREY, JAMES (1977). Sociology and Public Affairs: The Chicago School. Beverly Hills, California, Sage Publications.

CEFAI, DANIEL (2001). "Naturalism in American Sociology at the Turn of the Century: The Origins of the Chicago School Perspective", en La Revue du MAUSS, 17, pp: 261-274.

CHAPOUliE, JEAN-MiCHEL (1987). "Everett C. Hughes and the Development of Fieldwork in Sociology", en Urban Life, 15, 3, pp: 259298.

CHAPOULIE, JEAN-MICHEL (2001). La tradition sociologique de Chicago. 18921961. Paris, Seuil.

CORTESE, ANTHONY (1995). "The Rise, Hegemony, and Decline of the Chicago School of Sociology, 1892-1945", en The Social Science Journal. 32, 3, pp: 235-254.

CRESSEY, PAUL (1932). The Taxi-Dance Hall. A Sociological Study in Commercialized Recreation and City Life. Chicago, University Press.

CRESSEY, PAUL (1983). "A Comparison of the roles of the 'sociological stranger' and the 'anonymous stranger' in field research", en Urban Life 12, 1, pp: 102-120.

DENZIN, NORMAN y LINCOLN, YVONNA (1994). Handbook of Qualitative Research. Sage Publications, Inc.

DENZIN, NORMAN (1995). "Stanley and Clifford: Undoing an Interactionist Text", en Current Sociology, 43, pp: $115-123$.

FARIS, ROBERT LEE (1967). Chicago Sociology: 1920-1932. San Francisco, Chandler Publishing Co.

FORNI, FLOREAL (1993). “Estrategias de recolección y estrategias de análisis en la investigación social", en: Forni, F. et al., Métodos cualitativos II. La práctica de la investigación. Buenos Aires, Centro Editor de América Latina.

GOLD, ROBERT (1958). "Roles in sociological field observation", en Social Forces, 36, pp: 217-213.

GOULDNER, ALVIN (1970). The Coming Crisis of Western Sociology. Londres: Heinemann.

HAMMERSLEY, MARTYN (1989). The dilemma of qualitative method. Herbert Blumer and the Chicago Tradition. London-New York, Routledge. 
Sobre la observación participante en la escuela de Chicago. Un análisis de las monografías fundacionales

HAMMERSLEY, MARTYN (1981). "Using qualitative methods", en Social Science Information Studies, 1, 4, pp: 209-220.

HAMMERSLEY, MARTYN y ATKINSON, PAUL (1994). Etnografía: métodos de investigación. Barcelona, Paidós.

HARVEY, LEE (1987). Myths of the Chicago School of Sociology. Aldershot, Avebury.

HOMAN, ROGER (1980). “The Ethics of Covert Methods", en British Journal of Sociology. XXI, 1, pp: 46-59.

KURTZ, LESTER (1984). Evaluating Chicago Sociology: A Guide to the Literature, With an Annotated Bibliography. Chicago, University of Chicago Press.

LEWIS, DAVIS y SMITH, RICHARD (1980). American Sociology and Pragmatism: Mead, Chicago Sociology and Symbolyc Interaction. Chicago, University of Chicago Press.

MATTHEWS, FRED (1977). Quest for an American Sociology: Robert E. Park and the Chicago School. Montreal, McGill-Queen's University Press.

NOCK, DAVID (2004). "The Myth about "Myths of the Chicago School" Evidence from Floyd Nelson House", en The American Sociologist, 35, 1, pp:63-79.

OBSERSCHALL, ANTHONY (1972). The Establishment of Empirical Sociology: Studies in Continuity, Discontinuity and Institutionalism. New York, Harper \& Row.

PIOVANI, JUAN IGNACIO (2007). “La Observación”, en: Marradi, Alberto; Archenti, Nélida. y Piovani, Juan Ignacio, Metodología de las ciencias sociales. Buenos Aires, Emecé.

PLATT, JENNIFER (1983). "The Development of the "Participant Observation" Method in Sociology: Origin Myth and History", en Journal of the History of the Behavioral Sciences, 19, 4, pp: 379-393.

PLATT, JENNIFER (1985). "Weber's Verstehen and the history of qualitative research: The Missing Link", en The British Journal of Sociology. 36, 3, pp: 448-466.

PLATT, JENNIFER (1992). "Acting as a switchboard: Mrs. Ethel Sturges Dummer's role in sociology", en The American Sociologist. 23, 3, pp: 23-36.

PLATT, JENNIFER (1994). "The Chicago School and firsthand data", en The History of Human Sciences. 7, 1, pp: 57-80. 
PLATT, JENNIFER (1996). A History of Sociological Research Methods in America, 1920 1960. Cambridge, Cambridge University Press.

PLATT, JENNIFER (1998). “Chicago methods: reputations and realities", en: Tomasi, Luigi (Ed.), The Tradition of the Chicago School of Sociology. Aldershot, Ashgate.

PLATT, JENNIFER (1995). "Research Methods and the Second Chicago School", en: Fine Gary (Ed.), A Second Chicago School? Chicago, University of Chicago Press.

ROCK, PAUL (1979). The Making of Symbolic Interactionism. Totowa, N.J., Rowman \& Littlefield.

SCHWENDINGER, HERMAN y SCHWENDINGER, JULIA (1974). The Sociologists of the Chair: A Radical Analysis of the Formative Years of North American Sociology (1883-1922). New York, Basic Books.

SICA, ALAN (2007). "Defining Disciplinary Identity: The historiography of U.S. Sociology", en: Calhoun, Craig (Ed.), Sociology in America. A History. Chicago, The University of Chicago Press.

TAYLOR, STEVEN y BOGDAN, ROBERT (1986). Introducción a los métodos cualitativos de investigación. Buenos Aires, Paidós.

THRASHER, FREDERIC (1927). The Gang: A Study of 1313 Gangs in Chicago. Chicago, University Press.

THRASHER, FREDERIC (1928). "How to Study the Boys' Gang in the Open", en Journal of Educational Sociology, 1, 5, pp: 244-254.

VASILACHIS, IRENE (1993). Métodos cualitativos I. Los problemas teórico-epistemológicos. Buenos Aires, CEAL.

VERHOEVEN, JEFF (1993). "An Interview with Erving Goffman", en Research on Language and Social Interaction. 26, 3, pp: 317-348. 